\title{
Effectiveness of Intermittent Compression and Decompression with Glide in Stage 3 of Knee Osteoarthritis
}

\author{
Bhutta AH*, Awan WA and Husnain N \\ Lecturer, Riphah International University, Islamabad, \\ Pakistan \\ Vice Principal Isra University Islamabad, Pakistan \\ Physical Therapist, Pakistan \\ *Correspondling author: Abdul Haseeb Bhutta, \\ Lecturer, Riphah International University, Islamabad, \\ Pakistan
}

Received: July 27, 2018; Accepted: August 23, 2018; Published: August 30, 2018

\begin{abstract}
Objectives: Purpose of this study was to identify the effectiveness of mobilization (compression and decompression with glide) in patients with STAGE 3 osteoarthritis at knee joint.

Methodology: A total of 30 patients visiting the physical therapy department of Benazir Bhutto Hospital Rawalpindi Pakistan were enrolled in this study. The study design was Randomized controlled trail, patients were randomly assigned into two groups by using lottery method, Non probability convenience sampling technique was used in this study. All the patients were satisfying the inclusion and exclusion criteria. Group A was experimental group in which Intermittent compression and decompression with glide was given along with conventional Physical therapy intervention while in Group B only conventional physiotherapy interventions were given in the study to check the effectiveness of intermittent compression and decompression with glide in stage 3 of osteoarthritis at knee joint. Duration of the study was approximately 6 months. The data was collected through the following questionnaire/forms. Self-structured General demographic questionnaire and KOOS (knee injury and osteoarthritis outcome score) were used.

Results: Statically analysis of the data stated that in this study $27 \%$ were males and $73 \%$ females were included in this study. The mean age of patients were $51.40 \pm 5.9$ years. Average mean of height of patients was $160.1 \pm 9.5$ $\mathrm{cm}$, average mean weight was $75 \pm 9.1 \mathrm{~kg}$. The result of BMI shows that $37 \%$ were normal weighted while $63 \%$ were over weighted. Further investigation suggested that the total pain score at baseline of experimental group was 15.86 \pm 10.68 and in the end of sessions score was enhanced to 89.0 with P-value 0.0001 . Total symptom score of experimental group was $45 \pm 12.4$ and after end session the score was $85 \pm 8.3 \mathrm{P}$-value $0.001 \mathrm{ADL}$ score at baseline was 13.00 which enhance to 85 after 4 week of sessions with $p$ value of 0.001 . the mean score of QOL was $7.6 \pm 7.3$ enhanced to $93.5 \pm$ with p-value 0.001 which shows that this way of treatment have very significant role in regeneration of cartilage of knee joint which was degeneratively changed during the process of osteoarthritis which was elaborated by reduction of pain with increased Range of motion and improved quality of life.
\end{abstract}

Conclusion: Intermittent compression and decompression with glide has significant improvement in associated symptoms of osteoarthritis along with the advancement and improvement of quality of life. This technique can delay or prevent surgical intervention. This is a cost effective way of treatment of knee osteoarthritis and have better results then other treatments available.

Keywords: Osteoarthritis; Manual mobilization; Intermittent compression and decompression with glid; Koos 1.0

\section{Introduction}

Osteoarthritis (OA) is a degenerative joint disease, occurring primarily in older persons, characterized by erosion of the articular cartilage, hypertrophy of bone at the margins. Osteoarthritis $(\mathrm{OA})$ is the most common joint disorder in all over the world. Its occurs most in women of age over 45 . It occurs in $80 \%$ of people over 55 years of age, 23\% experience limitation of activities, Radiographic evidence of osteoarthritis is present in the majority of people over age $65 ; 80 \%$ of those over 75 , Approximately $11 \%$ of those over 65 have symptomatic osteoarthritis of the knee [1,2].

Damaged articular cartilage has less healing capacity due to its low metabolic activity and limited blood circulation which permit limited response to injury. This cartilage lesion is sometime very progressive. Therefore, its early intervention is aimed to limit this progressive lesion to articular cartilage which is may be critical for decreasing the disability and chronic pain associated with osteoarthritis. Compressive 
forces applied on the knee joint pushes the fluid out of cartilage and during decompression it is reabsorbed, this phenomenon helps in the regeneration of cartilage by means of supplying nutrients and oxygen. Physical therapy interventions included are electrotherapy along with knee isometric exercises for management of osteoarthritis. But these interventions are not so effective and do not help in regeneration of the cartilage. Intermittent compression and decompression with glide helps to activate osteoplastic activity and improve the symptoms of osteoarthritis thus delaying the process of degeneration.

\section{Methodology}

A total of 30 patients visiting the physical therapy department of Benazir Bhutto Hospital Rawalpindi Pakistan were enrolled in this study. The study design was Randomized controlled trail, patients were randomly assigned into two groups by using lottery method, non probability convenience sampling technique was used in this study. All the patients were satisfying the inclusion and exclusion criteria. Group A was experimental group in which Intermittent compression and decompression with glide was given along with conventional Physical therapy intervention while in Group B only conventional Physiotherapy interventions were given in the study to check the effectiveness of intermittent compression and decompression with glide in stage 3 of osteoarthritis at knee joint Duration of the study was approximately 6 months. The data was collected through the following questionnaire/forms. Self-structured General demographic questionnaire which consist of Name, age, gender date, height, weight, BMI marital status, Occupational history including types of job, health history including other disease/injury and osteoarthritis related information were asked in KOOS (knee injury and osteoarthritis outcome score). The intervention period for each patient was one month and data was collected on $1^{\text {st }}$ day, end of $2^{\text {nd }}$ week, end of $3^{\text {rd }}$ week and at the end of month. For between groups cross sectional comparison, Independent sample t-test was used and for within the groups longitudinal comparison repeated measure analysis of variance (RM-ANOVA) was used. The data was analyzed through SPSS 21.

\section{Results}

Statically analysis of the data stated that in this study $27 \%$ were males and $73 \%$ females were included in this study. The mean age of patients were $51.40 \pm 5.9$ years. Average mean of height of patients was $160.1 \pm 9.5 \mathrm{~cm}$, average mean weight was $75 \pm 9.1 \mathrm{~kg}$. The result of BMI shows that $37 \%$ were normal weighted while $63 \%$ were over weighted. Further investigation suggested that the total pain score at baseline of experimental group was $15.86 \pm 10.68$ and in the end of sessions score was enhanced to 89.0 with $\mathrm{P}$-value 0.0001 while the control group score was $14.8 \pm 12.8$ which enhance to $90.2 \pm 2.1$ having P-value 0.001 both group shows significant improving. Total symptom score of questionnaire KOOS's experimental group was $45 \pm 12.4$ and after end session the score was $85 \pm 8.3 \mathrm{P}$-value 0.001 and mean score of control group was $42 \pm 9.6$ enhance to $64 \pm 10.1$, having P-value $0.001, \mathrm{ADL}$ score at baseline was $13.00 \pm 10.03$ which enhance to $85 \pm 7.6$ after 4 week of sessions with $\mathrm{p}$ value of 0.001 and mean ADL score of control $14 \pm 10$ and enhance to $66 \pm 8$.6. The mean score of QO of experimental group was $7.6 \pm 7.3$ enhanced to $93.5 \pm 6.4$ with p-value 0.001 and the mean QOL score of control group was $8 \pm 6.7$ and enhances to $46 \pm 7.6$ which shows that this way of treatment have very significant role in regeneration of cartilage of knee joint which was degeneratively changed during the process of osteoarthritis which was elaborated by reduction of pain with increased Range of motion and improved quality of life.

\section{Discussion}

Few studies were available on this topic so it was a difficult task as limited literature was available. According to one study, during acute stage of osteoarthritis joint mobilization (compression and decompression with glide) results in significant improvement of the symptoms of the patient which support the results of our study. The initial effect of the (knee joint) mobilization on osteo-arthritic hyperalgesia.... [3]. The purpose of study was to find out the initial effects of accessory knee joint mobilization on pain management along with the functional abilities of patients with knee osteoarthritis. They analyse the effect of $9 \mathrm{~min}$ anteroposterior mobilization of TF joint (tibiofemoral) joint which was compared with manual contact and non contact interventions, the result of study explains that effect of manual mobilization (intermittent compression and decompression with glide) applied at knee joint help in reduction of pain and enhancement of functional capability with in the patients of knee joint osteoarthritis.

The study of Effectiveness of manual physical therapy and exercise in osteoarthritis of the knee by researcher suggested that manual mobilization (compression and decompression with glide) and supervised exercises for osteoarthritic patients yields in functional benefits in Activity of Daily Livings along with delaying or prevention from surgical interventions.

Another study "The Effect of Nutritional Supplementation and Exercise on Subjective Pain and Function in People with Osteoarthritis of the Knee" suggest that Intermittent compression and decompression along with gliding of articular cartilage allows the synovial fluid to nourish the joint and remove waste products present with in it, This movement can also lead to increase resilience and thickening of the cartilage. Manual mobilization along with exercises leads to increased muscle and ligamentous strength that helps in the stability to the joint, as well as decreasing joint pain along with other symptoms associated with osteoarthritis. This study explains that the group who were given intermittent compression and decompression with glide with nutritional supplement their symptoms of osteoarthritis was reduced and quality of their life was enhanced as compared to those group who were not given manual mobilization and exercise plan [4].

According to our studies this manual mobilization techniques improves the quality of life and reduce the symptoms and pain associated with osteoarthritic effect thus indicating that intermittent compression and decompression only regenerate the cartilage of knee joint but do not have significant improvement in ligamentous injuries. Further studies should be conduct with arthroscopy to investigate that in a real sense how much regeneration is achieved with this technique.

Implementation of this technique can reduce and avoid the surgical intervention as it is very expensive treatment and healing chances of knee joint is not that satisfactory. This technique is cost effective as well as most significant in reducing symptoms of 
osteoarthritis at knee joint as compared to surgery and intraarticular injection. Majority of geriatric population within Pakistan is suffering from osteoarthritis specially females above 40 due to post menopausal effect. Decrease in oestrogen level in blood leads to weakness of bones and degeneration of cartilage. The onset of osteoarthritis have worst effect physically psychologically and socially [4].

\section{Conclusion}

This mode of treatment was more efficient and shows good result in $\mathrm{x}$-rays and improved quality of life measure by Knee injury and Osteoarthritis Outcome Score (KOOS), English version LK1.0. It helps in the regeneration of cartilage, reduces the pain and enhances the ROM while making easy for the patient to perform daily life routine work. The majority of a Geriatric population is suffering from osteoarthritis of knee joint resulting in psychologically, socially and functionally disabled. This prevents them from their participation as an active member of the society. Manual Mobilization (intermittent compression and decompression with) is an effective way of reducing their disability and keeps them pain-free and functionally active. This also helps in or prevention and delaying the need for surgical procedures but further research and evidence are necessary for the efficacy of manual mobilization techniques in osteoarthritis of the knee. In last, mobilization helps in a great way for management of pain, enhancement of a range of motion and improvement of the functional abilities of the patient diagnosed with osteoarthritis.

\section{Limitation \& Recommendation}

A small sample size was used this was due to lack of referral of patients of acute and sub acute stages of OA to the OPD of physical therapy. In addition, our study design had no control or comparison group. Further studies should be conducted to find out arthroscopic evaluation about this technique to investigate cartilage regeneration, $\mathrm{x}$-ray and MRI reports should b checked for efficacy of this technique at knee joint.

\section{References}

1. Bandy W. Sanders B. Therapeutic exercise for physical therapist assistants. Philadelphia: Lippincott, Williams \& Wilkins. 2007.

2. PP-DPT IR, Muhammad Naveed Babur PT DP. The effectiveness of early mobilization (compression and decompression with glide) in osteoarthritis of knee joint. Interdisciplinary Journal of Contemporary Research In Business. 2012; 3: 252.

3. Power A, Scheffer D. The effect of nutritional supplementation and exercise on subjective pain and function in people with osteoarthritis of the knee. 1999.

4. Porcheret $\mathrm{M}$, Jordan $\mathrm{K}$, Croft $\mathrm{P}$. Treatment of knee pain in older adults in primary care: development of an evidence-based model of care. Rheumatology. 2007; 46: 638-648.
Phys Med Rehabil Int - Volume 5 Issue 4 - 2018

ISSN : 2471-0377 | www.austinpublishinggroup.com

Bhutta et al. (C) All rights are reserved
Citation: Bhutta AH, Awan WA and Husnain N. Effectiveness of Intermittent Compression and Decompression with Glide in Stage 3 of Knee Osteoarthritis. Phys Med Rehabil Int. 2018; 5(4): 1151. 